\title{
Review
}

\section{Foraminal and far lateral lumbar disc herniations: surgical alternatives and outcome measures}

\author{
NE Epstein*,1,2,3 \\ ${ }^{1}$ The Albert Einstein College of Medicine, Bronx, NY, USA; ${ }^{2}$ Department of Neurosurgery, The North Shore-Long \\ Island Jewish Health System, Manhasset, NY 11030 and New Hyde Park, NY 11040, USA; ${ }^{3}$ Long Island \\ Neurosurgical Associates, PC, New Hyde Park, NY 11040, USA
}

Far lateral disc herniations constitute $7-12 \%$ of all disc herniations. They may be purely far lateral or extraforaminal in location, located beyond the pedicles, or may include intraforaminal and even intracanalicular components. Occurring predominantly at the L4L5 and L3 - L4 levels in almost equal numbers, they are occasionally noted at L5-S1. Clinical syndromes reflect compression of the superiorly exiting nerve root and ganglion; ie an L4-L5 far lateral disc produces a L4 root syndrome. Clinical complaints often include severe radicular pain accompanied by very positive mechanical signs; Laségue and reverse Laségue (femoral stretch test) maneuvers. Neurological deficits, including motor, reflex, and sensory findings, are seen over $75 \%$ of the time. Although conservative management is occasionally successful (10\%), surgery is usually required. The extent of stenosis and attendant degenerative changes dictate whether laminectomy, hemilaminectomy or laminotomy are required along with one of several facet resection options; full facetectomy, the intertransverse approach, medial facetectomy, or an extreme lateral procedure. Postoperatively, patients' neurological outcomes based on both surgeon and patient based outcome measures (SF-36), were comparable for the different surgical procedures which had been based on the individual patient's pathology.

Spinal Cord (2002) 40, 491-500. doi:10.1038/sj.sc.3101319

Keywords: lumbar foraminal discs; far lateral discs

\section{Introduction}

Four major facet resection techniques are employed for removal of far lateral disc herniations (FLD). These include the medial facetectomy, the intertransverse approach with preservation of the pars interarticularis and mid-portion of the facet joint, the full facetectomy, and the extreme lateral approach. The extent of coexistent spinal stenosis mandates differing types of accompanying decompression techniques. Laminotomy suffices in the absence of spinal stenosis, the hemilaminectomy is necessary for more extensive but focal disease, and the total laminectomy for spinal stenosis, spondyloarthrosis, degenerative spondylolisthesis, degenerative scoliosis, limbus vertebral fractures, and any combination thereof. No medial dissection accompanies the extreme lateral approach. The extent and type of facet resection and decompression employed to approach far lateral disc herniations must be individualized as no one technique is universally appropriate. ${ }^{1}$

*Correspondence: NE Epstein, Long Island Neurosurgical Assoc., P.C., 410 Lakeville Rd. Suite 204, New Hyde Park, NY 11042, USA
The clinical, neurodiagnostic, surgical management, and outcomes utilizing the Short-Form 36 questionnaire in patients undergoing far lateral disc surgery are reviewed.

\section{Materials and methods}

\section{Anatomy}

Computed tomographic evaluation of the far lateral compartment of 200 cadaver specimens demonstrated that the interpedicular space extends further laterally as the pedicles increasingly diverge moving caudally in the lumbar spine. ${ }^{2}$ In the upper lumbar spine, the interpedicular space is minimal and the facet joint covers more of the disc space and neural foramen, decreasing the available space for disc removal and necessitating more extensive sacrifice of the medial facet for exposure. Extensive bony removal with partial or complete facetectomy, and greater degrees of pars resection are necessary to adequately expose upper lumbar foraminal and far lateral pathology. At the L5 and S1 level the interpedicular diameter is widest and 
minimal to no facet removal is needed to uncover the foraminal or far lateral compartment.

Microdissection of cadavers and spinal specimens, and experience clinically with over 200 patients, Schlesinger, Frankhauser and de Tribolet again confirmed that as one extends caudally, more and more bone covers the intervertebral foramen allowing for a diminished available work space in the far lateral compartment. $^{2}$ This therefore necessitates bone removal from the lateral portion of the pars interarticularis and superolateral aspect of the facet joint at the lower lumbar levels. Exposure then provides for direct visualization of the dorsal root ganglion.

Cadaveric dissections of the lower lumbar spine using sagittal sections combined with cadaveric biomechanical studies revealed four distinct intraforaminal ligaments. ${ }^{3}$ Four bands extend radially from the nerve root sleeve, the first being found at the facet capsule posteriorly, two attaching to the superior and inferior pedicles, and the fourth to the disc anteriorly. ${ }^{3}$

\section{Definition of the far lateral compartment}

The 'far lateral' compartment is anatomically defined as the area lateral to the superior and inferior pedicles, with the disc situated anteriorly and the vertebral body and leading edge of the superior articular facet medially with the facet joint itself found dorsally. Compromise of a nerve root far laterally may be acutely exacerbated when far lateral stenosis exists and is accompanied by degenerative disease: spondyloarthrosis, degenerative spondylolisthesis scoliosis, and limbus vertebral fractures.

\section{Frequency and location of far lateral discs}

Far lateral discs represent $7-12 \%$ of all lumbar disc herniations and usually involve free fragments which have migrated superolateral to the disc space of origin. ${ }^{1,4-10}$ Purely foraminal lesions, found subarticularly bordered by the medial and lateral superior pedicle, occur in $3 \%$ of disc herniations, while another $4 \%$ exhibit intra and extraforaminal extension. ${ }^{11}$ These lesions compromise the superiorly exiting nerve root and ganglion, culminating in superior nerve root syndromes. Disc herniations at $\mathrm{L} 1-\mathrm{L} 2$ produce a deficit in the L1 root distribution, while L2-L3 discs yield L2 root deficits, L3-L4 discs contribute to L3 root complaints, L4-L5 discs produce L4 deficits, and L5-S1 lesions are responsible for L5 root compromise.

Most frequently far lateral disc herniations are encountered at either the L3-L4 or L4-L5 levels followed by L5-S1. ${ }^{1,5,9,12,13}$ Cephalad involvement of $\mathrm{L} 1-\mathrm{L} 2$ or $\mathrm{L} 2-\mathrm{L} 3$ is rare except in An et al's study which noted an unusually high incidence of $28 \%{ }^{4}$

\section{Clinical and neurological parameters}

Patients with far lateral discs are typically in their midfifties, ranging from $50-78$ years of age. ${ }^{1,4,12}$ Males and females present in equal numbers. Patients typically demonstrate extreme radicular pain associated with compromise of the dorsal nerve root ganglion in the far lateral compartment. Leg pain is usually unremitting and back pain is often minimal. Patients with more cephalad far lateral discs exhibit proximal hip pain radiating into the thigh and knees. Neurological signs for far lateral discs involving the $\mathrm{L} 1-\mathrm{L} 2$ through the L4-L5 levels may include a positive reversed Laségue maneuver or femoral stretch test, iliopsoas and/or quadriceps weakness (L1-L4 roots), diminished to absent patellar responses and proximal sensory changes. Far lateral discs in more cephalad locations must be differentiated from hip and knee pathology with appropriate X-ray and MR studies, while arterial dopplers may determine the existence and/or coexistence of vascular claudication. The most distal far lateral discs, found at the L5-S1 level with L5 root impingement result in severe sciatic syndromes often characterized by a positive Laségue sign, weakness of the extensor hallicus longus and dorsiflexors, diminished or absent Achilles response, and decreased pin appreciation along the L5 distribution.

\section{Other pathology contributing to far lateral root compromise}

\section{Limbus vertebral fractures}

Fractures of the vertebral limbus alone or in combination with lateral, foraminal or far lateral stenosis and/ or disc disease may result in marked far lateral nerve root compression. ${ }^{14,15}$ Four types of limbus fractures have been identified. Type I fractures are cortical 'shelf' fractures which span the width of the disc space compromising the central spinal canal and extending into the lateral recess and proximal foramina. Type II lesions include both cortical and cancellous elements with midline impingement directly on the central thecal sac. Type III fractures include lateral non-ossified cartilaginous (III A) or ossified cortical (III B) fractures of the vertebral end plates originating from the cephalad or caudad vertebral body. Type IV limbus fractures involve the full sagittal length of the posterior vertebra spanning from interspace to interspace, and more frequently contribute to cauda equina syndromes.

Resection of limbus vertebral fractures associated with foraminal or far lateral root compression, often requires a full facetectomy with laminectomy to adequately expose the nerve root over its entire course. ${ }^{14}$ Resection first requires emptying out the underlying disc space to create a trough. The limbus fracture is then morcellated with a downbiting curette, tamp and mallet technique. Fragments are initially delivered inferiorly into the interspace, and then excized in a piece-meal fashion to accomplish the decompression.

Stenosis and spondylosis

Lumbar spinal stenosis may accompany far lateral disc pathology. ${ }^{3,6,15}$ Older patients demonstrated significant 
accompanying stenosis on myelo-CT studies (72\%), and surgical procedures had to be tailored to address the greater extent of disease. ${ }^{12}$ An et al's 50 patients with far lateral discs with stenosis required more extended decompressions. ${ }^{4}$ In 857 patients having surgery for lumbar spinal stenosis, 45 exhibited far lateral pathology including 40 far lateral discs and five instances of far lateral stenosis, spondylosis, degenerative spondylolisthesis, scoliosis, and limbus fractures. ${ }^{6,7,15}$

Short vertically oriented pedicles, thickened lamina, massive arthrotic facets, and other degenerative changes contribute to diffuse stenosis increasing the challenges of far lateral disc surgery. Decompression of minimal focal stenosis may require only a laminotomy or hemilaminectomy with medial facetectomy or intertransverse approach, while multilevel laminectomy with full facetectomy may be required to address multilevel disease. Intraoperative localizing films, including one with a Penfield elevator in the interspace, should be performed in all procedures. Subsequent fusion may be considered.

\section{Degenerative spondylolisthesis}

Grade I degenerative spondylolisthesis is typically found at the L4-L5 level, followed by L3-L4, L2L3, and the L5-S1 levels respectively. ${ }^{9,10,16}$ Olisthy, limited to one-quarter of the vertebral body, is generally attributed to a locking of sagitally-oriented, hypertrophied posterior facet joints. ${ }^{16}$ Disc herniations accompany degenerative spondylolisthesis in $4.3-20 \%$ of patients. Far lateral disc herniations occurring at the level of a slip typically require full unilateral facetectomy with an instrumented fusion to avoid instability in patients under 65 years of age, while select patients over the age of 65 , may alternatively be addressed with a more restricted in situ posterolateral intertransverse fusion. ${ }^{1,9}$ Good to excellent results are cited in up to $80 \%$ of patients.

\section{Spondylolisthesis with spondylolysis}

Spondylolisthesis with spondylolysis compromises the nerve root as it enters and continues within the neural foramen, maximal compression occurs beneath the mobile defect in the pars interarticularis. An additional fragment in this area will not be well-tolerated. Surgical extirpation of the entire inferior articular facet including the lysis defect is required to free up the nerve root along its entire intracanalicular, foraminal, and extreme lateral course. Patients with spondylolisthesis with lysis typically require simultaneous instrumented fusion and consideration of posterior lumbar interbody fusion, with good to excellent outcomes produced in $80-85 \%$ of reported cases. ${ }^{10}$

\section{Degenerative scoliosis}

Older patients with degenerative scoliosis often demonstrate MR and CT evidence of foraminal or far lateral comprise of exiting nerve roots, particularly on the 'inside' of the ' $\mathrm{S}$ ' shaped curves. Rotational and conformational deformity may lend to an exaggeration of the degree of root compromise and, therefore, neurological and radiological findings must be carefully correlated. Far lateral lesions may respond to unilateral decompression alone, but more typically, unilateral decompression should be accompanied by bilateral fusion.

\section{Neurodiagnostic evaluation}

\section{Magnetic resonance scans (MR)}

Far lateral soft disc herniations located lateral to the pedicles appear as isointense to hypointense lesions. Where hypointense fat normally surrounds the dorsal root ganglion, a loss of fat may signal a disc herniation. Parasagittal MR studies yield the most direct view of the neural foramen and far lateral compartment, and clearly demonstrate soft discs, however, bony pathology appearing as a diffuse hypointense mass is less exact. Therefore, CT scans should also be performed to define and demonstrate the extent of bony disease.

MR studies with Gadolinium enhancement (Diethylenetriamine-Pentaacetic Acid), may differentiate tumor (ie; neurofibroma, metastatic tumor-enhances) from a sequestrated far lateral disc (non-enhancing). ${ }^{17}$ Postoperative, enhanced MR studies may also distinguish between scar and recurrent disc herniations. ${ }^{18}$

\section{$C T$ and Myelo-CT scans}

Computed tomography aids in the diagnosis of far lateral disc herniations (Figures 1-4). ${ }^{17,19}$ Isolated far lateral fragments appear isointense, while contralaterally the normal dorsal root ganglion is surrounded by fat. Far lateral stenosis attributed to limbus vertebral fracture, dorsal facet arthropathy, or deformity may also be seen on varying CT examinations including 2-D or 3-D reconstructed images. Myelo-CT examinations readily demonstrate accompanying spinal stenosis situated centrally, laterally, or within the proximal neural foramen. However, CSF pathway impingement cease beyond the proximal neural foramen where the arachnoid root sleeve ends. Myelo-CT findings may alter the operative technique utilized in older patients $(72 \%)$ with accompanying stenosis. $^{12}$ Rarely, CT discography may demonstrate a far lateral lesion by revealing extravagation of dye far laterally.

\section{Conservative management}

Patients with far lateral discs may respond to conservative management consisting of steroidal and non-steroidal anti-inflammatory medication. Rust and Olivero found that 12 of $17(71 \%)$ patients with far lateral discs did not require surgery. ${ }^{20}$ Weinder and 


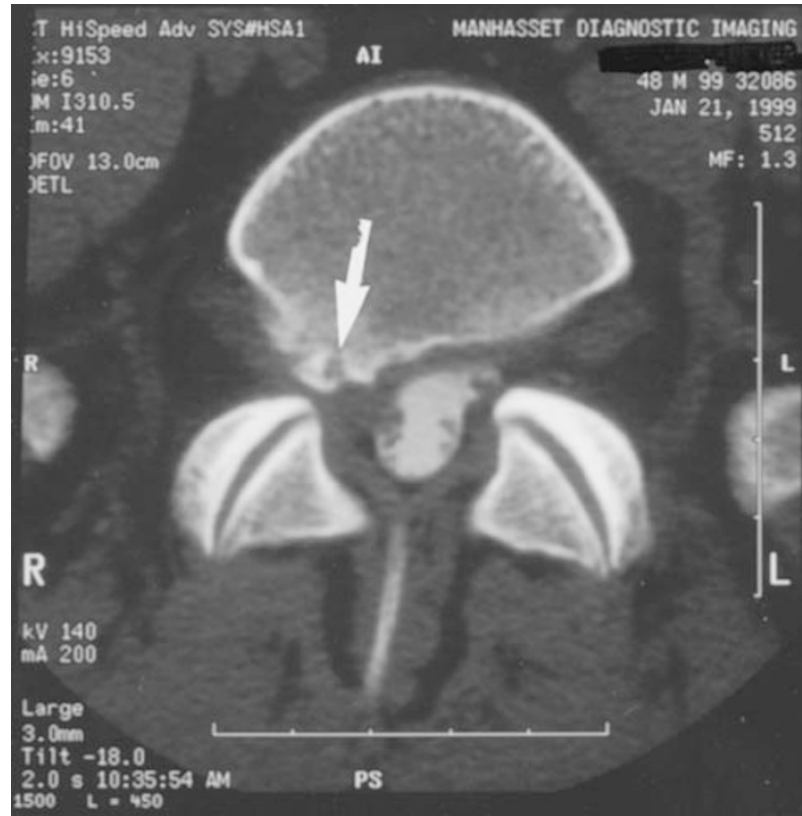

Figure 1 Type III calcified limbus vertebral fracture occupying the proximal and mid portion of the right L4L5 neural foramen (arrow)

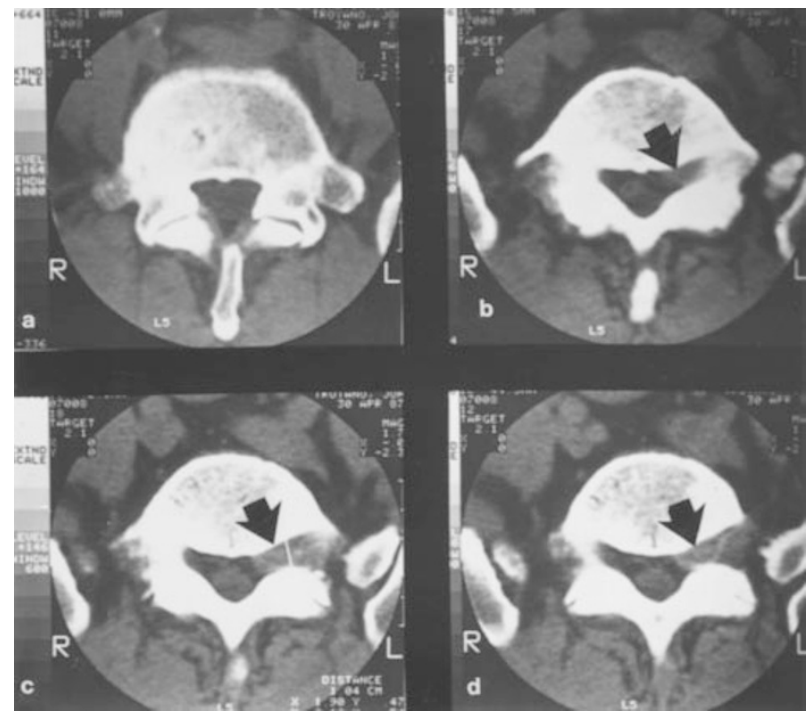

Figure 2 (a) Non contrast CT scan obtained at the mid L5 vertebral level. (b) Non-Contrast CT scan at the upper level of the L5-S1 interspace showing disc material extending laterally, foraminally, and far laterally into the neural foramen (arrows). (c) The CT study at the mid foraminal level most clearly demonstrated disc extending from the lateral recess all the way to and through the far lateral compartment (arrows). (d) This CT image, obtained at the lower-most aspect of the L5-S1 neural foramen, showed foraminal and far lateral disc (arrows)

Fraser observed that trans-foraminal injections of local anesthesia and steroids provided immediate relief based on their analysis of the Low Back Outcome Score in 27

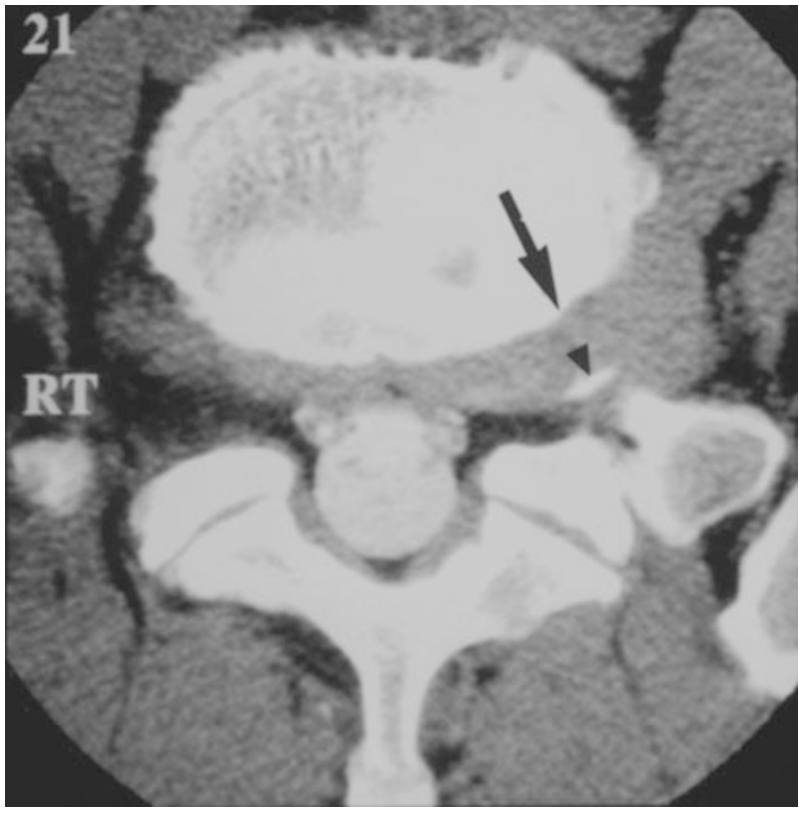

Figure 3 Transaxial myelo-CT scan revealed a Grade I spondylolisthesis (arrow) at the L5-S1 level combined with a left-sided foraminal and far lateral disc herniation (arrows)

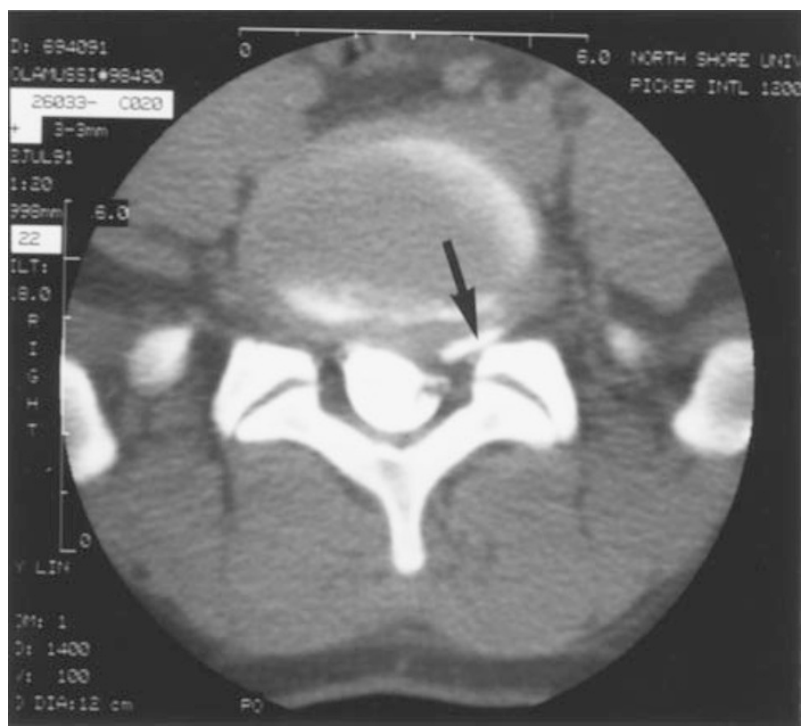

Figure 4 The myelo-CT scan at the L5-S1 level demonstrated a lateral, foraminal and far lateral left-sided disc herniation in conjunction with a Type III 'lateral chip' limbus vertebral fracture (arrows)

out of 30 patients and long-term relief in 22 of 28 patients, while three required surgery. ${ }^{21}$ Other studies indicate $10 \%$ of patients were managed successfully without surgery. ${ }^{1,13}$ The presence of significant neurological deficits should, however, prompt the examiner toward more rapid consideration of surgical alternatives. 


\section{Different facet resection techniques}

\section{Medial facetectomy}

The medial facetectomy with laminotomy or hemilaminectomy may provide adequate access to proximal foraminal lesions, particularly at the L5-S1 level (Figures 5 and 6). However, such limited foraminal exposure rarely suffices in the presence of coexistent degenerative changes such as spondyloarthrosis, degenerative spondylolisthesis, and scoliosis. Also, foraminal dissection utilizing angulated downbiting curettes or Woodson dissectors may result in injury to the foraminal nerve root.

\section{Intertransverse technique (ITT)}

An extended interlaminar laminotomy or hemilaminectomy combined with both medial facetectomy/ foraminotomy and lateral extraforaminal exposure provides for preservation of the intervening mid portion of the facet joint and pars interarticularis (Figure 7). ${ }^{22}$ Medially, visualized is the thecal sac and proximal exiting nerve root affording adequate access to the medial disc and central, lateral, or proximal foraminal stenotic lesions. Extraforaminal exposure will become increasingly limited as one extends to further caudal levels. The extraforaminal compartment is bordered by the pedicles superiorly and inferiorly, the disc anteriorly, the yellow ligament and facet joints posteriorly, and a fat bed laterally. Where significant foraminal or far lateral stenosis is present the attempt to employ the intertransverse techniques may risk inadvertent neural injury. In such cases, a full facetectomy safely exposes the nerve root while avoiding a 'blind' dissection; selective stabilization may be required.

Advantages of the ITT procedure includes both medial and far lateral exposure. This affords access to medial stenosis and central disc herniations; the former limiting the risk of recurrent radiculopathy secondary to exacerbation of stenosis by disc resection, and the latter reducing the potential for disc recurrence. ${ }^{22,23}$ Radiographic documentation of the extent of facet resection performed in conjunction with far lateral disc resection of $<50 \%, 51-74 \%$ or $75-100 \%$ did not appear to significantly alter the frequency of instability encountered in one study with the interlaminar approach providing adequate far lateral exposure. ${ }^{23}$

\section{Full facetectomy}

Spondylosis, arthrosis, degenerative spondylolisthesis, scoliosis, far lateral stenosis, and limbus fractures may necessitate complete facetectomy to approach far lateral pathology (Figure 8). Full facetectomy offers the lowest incidence of retained disc fragments, is the most familiar approach, and therefore limits inadvertent neural trauma. Clinically symptomatic instability is relatively infrequent. Fusion was required in only one of 41 of Garrido and Connaughton's series of

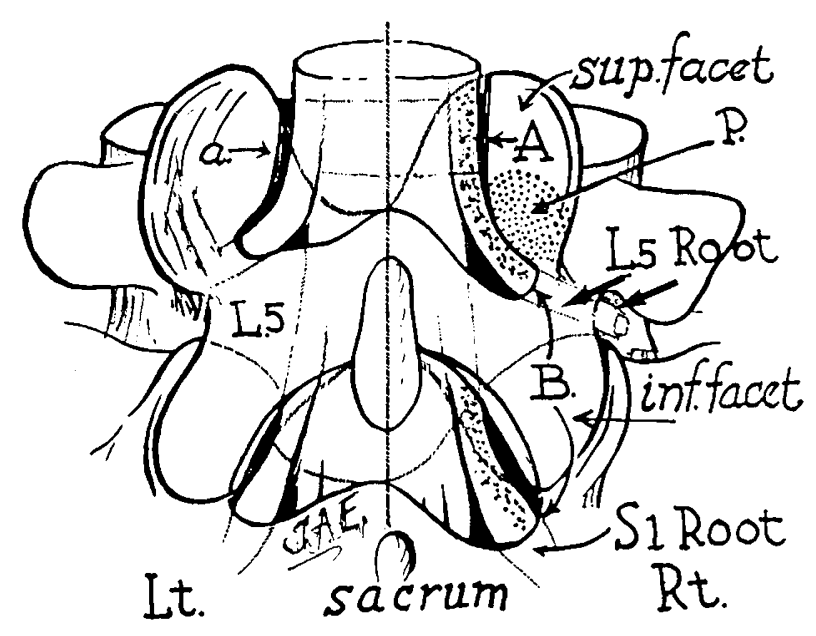

Figure 5 Illustration following coronal hemilaminectomy of the superior aspect of L5 exposing the L5 root on the left side (a) and right side (A) following a medial facetectomy with foraminotomy $(B)$

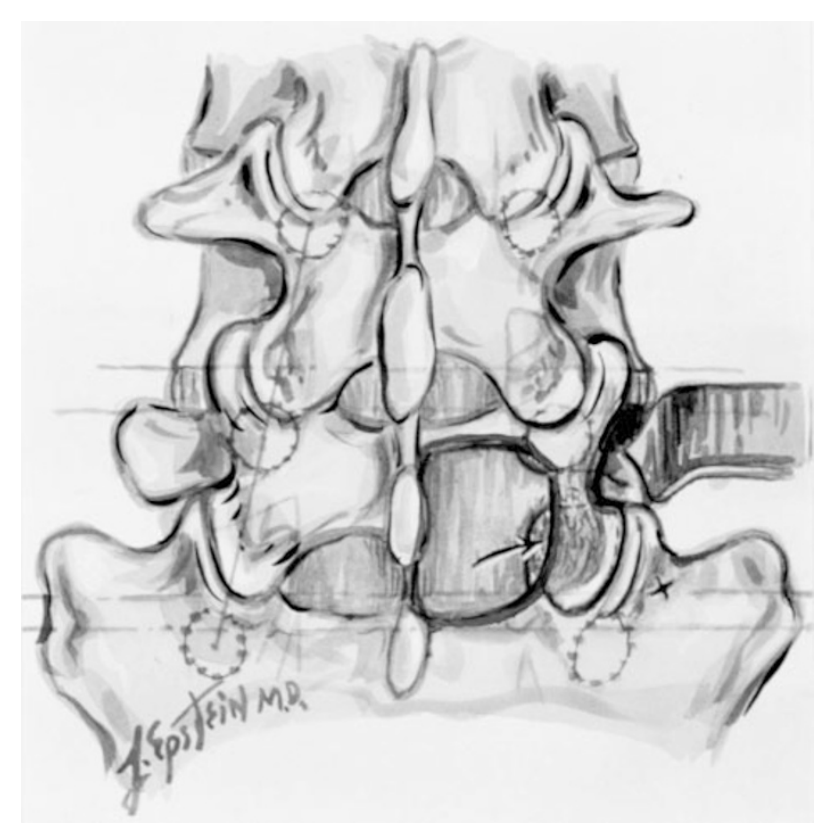

Figure 6 Medial facetectomy, foraminotomy and extended right L5 laminotomy for exposure of the L5 nerve root (arrow) as it extends into the proximal L5-S1 neural foramen

patients undergoing far lateral disc surgery including full facet resection. ${ }^{19}$ In Epstein's initial series of 60 patients with far lateral discs, only one required a secondary fusion, while only four of 170 patients with far lateral disc herniations and degenerative spondylolisthesis at the same level required subsequent fusions. ${ }^{12}$ It should be noted that at L5-S1, the iliotransverse ligament provides excellent support if the L5 vertebra is located below the intercrestal line. 

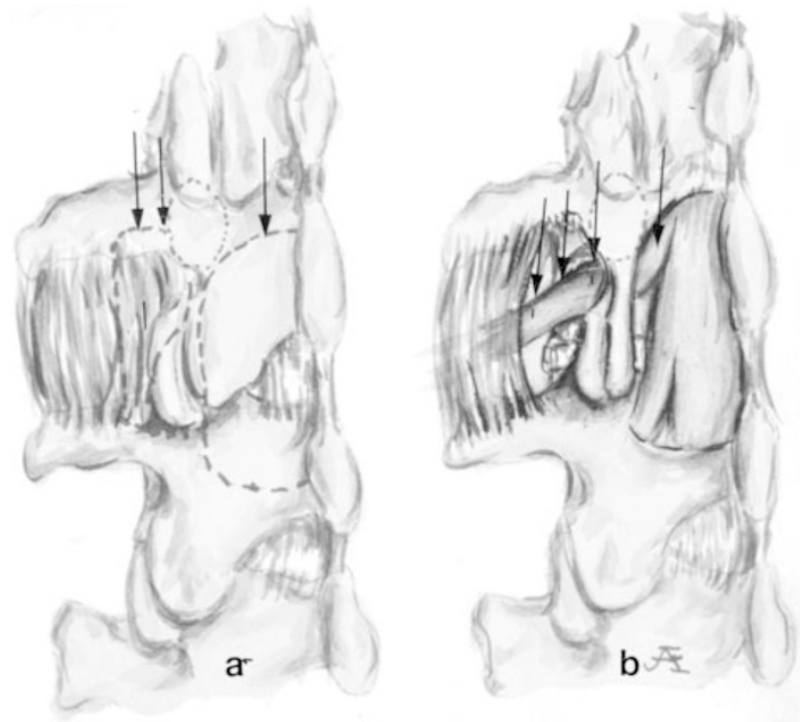

Figure 7 (a) On this left lateral view of the lumbar spine at the L4-L5 level, the dotted lines delineate the medial (single arrow) and lateral (double arrows) exposure which constitute the intertransverse approach. (b) The intertransverse approach at the L4-L5 level on the left side included a medial facetectomy with foraminotomy for exposure of the L4 root (single arrow) within the spinal canal, followed by extraforaminal exposure (triple arrows)

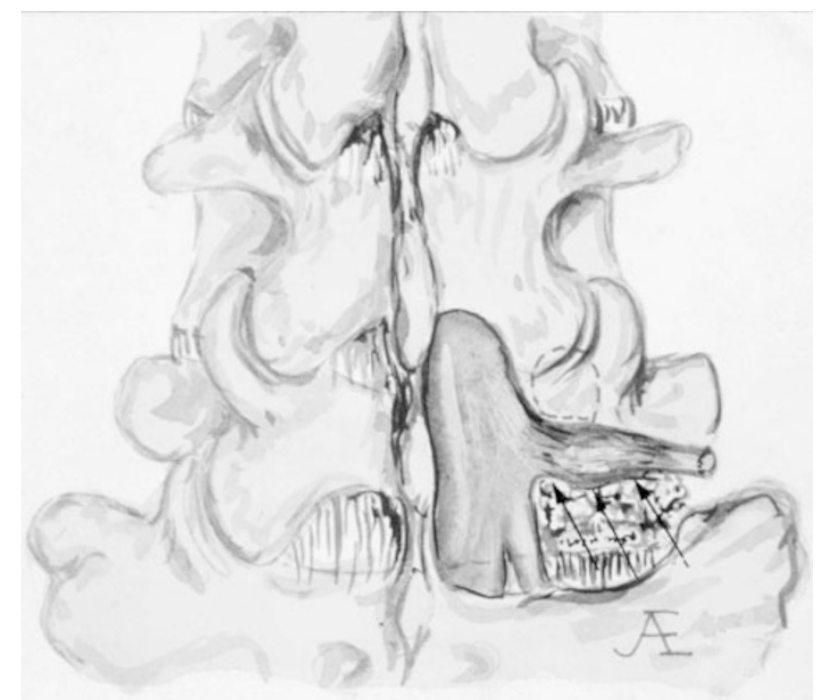

Figure 8 On the right at the L5/S1 level, a full facetectomy requires an ipsilateral L5 hemilaminectomy with S1 laminotomy, and full resection of the L5 inferior articular facet. The entire course of the lateral, foraminal, and far lateral L5 root (arrows) is exposed, allowing for resection of any type of lateral, foraminal and far lateral pathology

\section{Trans-pars technique}

The efficacy of the transpars approach to far lateral disc herniations is controversial and fraught with numerous deterrents. Initiated by a laminotomy at the level cephalad to the far lateral disc excision it requires that the far laterally exiting nerve root be isolated medially, followed laterally below the cephalad pedicle and foraminally beneath the pars and inferior articular facet, and finally far laterally. A major disadvantage is the elimination of access to the medial portion of the disc while compromising the facet joint thereby increasing the risk of instability. This approach, used in only one patient resulted in rapid disc recurrence and was deemed ineffective. ${ }^{1}$

\section{Extraforaminal (extreme lateral) approach}

The extreme lateral extraforaminal technique (EF) exposed through midline or preferably paramedian muscle splitting approaches, affords access only to the far lateral compartment and nerve root. ${ }^{11,19,24} \mathrm{Re}$ moval of the intertransversarious ligament and fascia adequately exposes the far lateral compartment at the L1 - L2, L2 - L3, and L3-L4 levels. The superolateral portion of the facet must also be removed to caudally identify the more medial portion of the far laterally exiting nerve root.

The exposure is appropriate for pathology confined to the far lateral compartment beyond the pedicles. ${ }^{11}$ Darden et al excised 25 far lateral discs using the muscle splitting approach and obtained a 2 year follow-up; $48 \%$ exhibited excellent, $32 \%$ good, $20 \%$ fair/poor results with surgery, low back pain and dysesthesias remaining the major complaints without radiographic evidence of instability. ${ }^{25}$ In Siebner and Faulhauer's series of 40 patients with far lateral lesions removed through 38 midline and two paramedian EF approaches, pain was successfully relieved in $85 \%$ of their patients. The minimal bony decompression and facet excision limited instability. ${ }^{11}$ In patients with prior surgery involving the medial spinal canal, the extraforaminal (EF) technique provides excellent exposure of far lateral pathology while avoiding epidural scarring. Limitations of the EF approach include the inability to remove disc medially, and the lack of access to foraminal and intracanalicular spondyloarthrosis.

\section{Endoscopic techniques}

Transforaminal arthroscopic and endoscopic techniques are also utilized to approach foraminal and far lateral disc herniations. In Kambin et al's study of 40 patients with lateral recess stenosis and a sequestrated foraminal disc having posterolateral arthroscopic procedures performed resulted in satisfactory outcomes in $82 \%$, few demonstrated postoperative causalgia secondary to painful manipulation of the dorsal nerve root ganglion. ${ }^{26}$ Ditsworth et al employing a transforaminal endoscopic diskectomy found 100 of 533 patients with far lateral discs exhibited a $91 \%$ success rate. ${ }^{27}$ They concluded that guidable endoscopes small enough to extend through the neural foramina would allow for excision of sequestrated fragments not limiting the use of this technique to 'contained' herniations. 
Anterolateral retroperitoneal approach

The anterolateral retroperitoneal approach to far lateral lumbar disc herniations requires a left-sided incision above the 12th rib, retroperitoneal dissection and the retraction of soft tissues including the psoas muscle, followed by exposure of the involved disc level. They may be supplemented with anterior lumbar interbody fusion utilizing either titanium cage devices or bone dowel techniques. Anterior approaches, particularly those performed laparoscopically, cause less perineural and peridural scar formation as most of the dissection is performed ventrally within the disc space, leaving the posterior elements undisturbed. Disadvantages include the inherent morbidity of a transabdominal retroperitoneal or laparoscopic procedure, with associated inability to clearly visualize the more medial portion of the involved nerve root thereby increasing the risk of retained or recurrent herniated disc fragments.

\section{Fusion}

\section{Fusion requirements}

The more facet removed in far lateral disc surgery, the greater the risk of instability. The extraforaminal exposure does not compromise the facet joint, the medial facetectomy removes the medial $25 \%$ of the facet joint, the intertransverse approach removes $25 \%$ of the medial and $25 \%$ of the superolateral facet joint thus preserving the pars interarticularis and mid 50\% of the facet, while the full facetectomy sacrificies $100 \%$ of the inferior articular facet.

Clinical data has demonstrated that even with full facetectomy, clinically symptomatic instability was rare. One of 41 patients in Garrido and Connaughton's series, and four of 170 in our experience required fusions. ${ }^{1,12,19}$ The advent of more formal prospective outcome studies has revealed more subtle postoperative pain syndromes and findings of 'micro-instability' warranting a higher frequency of this author's elective primary fusions in the younger population, particularly those who plan to return to labor intense occupations.

\section{Pedicle fixation}

Pedicle fixation for stabilization following far lateral disc excision, particularly where full facetectomy has been completed has been associated with limited morbidity. Younger patients, under 65 years of age, undergoing full unilateral facetectomy for far lateral disc excision should be considered as candidates for instrumented fusions including pedicle/screw/rod fixation techniques with the selected application of posterior lumbar interbody fusion devices.

\section{Outcomes}

Differing success rates are reported for far lateral disc surgery. An et al observed a $64 \%$ incidence of excellent and $28 \%$ frequency of good outcomes. ${ }^{4}$ Siebner and Deckler observed an $85 \%$ rate of good to excellent results following extraforaminal approaches to far lateral discs. ${ }^{11,25}$ We found no significant difference in outcomes for the three major facet resection techniques employed in far lateral disc surgery, 79, 70 and $68 \%$ exhibiting good to excellent outcomes after intertransverse, full facetectomy, and medial facetectomy procedures. ${ }^{1,13}$

\section{Far lateral disc procedures}

\section{Clinical data}

From 1984-1994, 170 of our patients had far lateral disc surgery and were followed an average of 5 years. ${ }^{1}$ Patients were typically 55 years of age, and included more males (112 patients) than females (58 patients). Most had subclinical symptoms for over 2 years, but almost all experienced subacute or acute deterioration within an antecedent 6 month period.

Physical findings included atrophy (31 patients) and extremely positive mechanical findings including ipsilateral knee contractures (143 patients), positive Lasegue maneuvers (159 patients), and positive femoral stretch tests (reverse Laségue maneuvers) (145 patients), motor deficits (126 patients), reflex abnormalities (167 patients), and radicular sensory deficits (135 individuals) were prominent, but these lesions were rarely associated with massive central canal compromise making a cauda equina syndrome with loss of bladder and bowel function rare (seven patients).

\section{Preoperative $M R$ and $C T$ studies}

Preoperative MR or CT based studies were always performed with the MR better demonstrating the soft tissue elements and CT studies directly revealing the extent of spondyloarthrosis, stenosis, scoliosis, and/or limbus vertebral fractures. Neurodiagnostically, 68 patients had far lateral discs at L4-L5, 63 at L3$\mathrm{L} 4,33$ at $\mathrm{L} 5-\mathrm{S} 1$, four at $\mathrm{L} 2-\mathrm{L} 3$, and two at $\mathrm{L} 1-\mathrm{L} 2$. Far lateral stenosis was also found in 30 patients, and degenerative spondylolisthesis was present in 23 .

\section{Flexion and extension $X$-rays}

Preoperatively and again 3 months postoperatively, dynamic films were performed to assess spinal instability. If $4 \mathrm{~mm}$ of motion was demonstrated or there was 'fish-mouthing' at the involved level, patients were deemed radiographically unstable.

\section{Surgical procedures}

In 36 of 170 patients, more severe lumbar spondyloarthrosis, stenosis, degenerative spondylolisthesis, and scoliosis accompanied far lateral disc disease. Multilevel laminectomies were therefore performed in 
conjunction with more extensive degrees of facet resection including full facetectomy in 58\%, intertransversectomy in $25 \%$, and medial facetectomy in $17 \%$. In the remaining 134 patients with mild to moderate stenosis, lesser degrees of facet resection were required including full facetectomy $(38 \%)$, medial facetectomy $(24 \%)$, and intertransversectomy $(37 \%)$.

A small subset of patients following far lateral disc surgery required second (25 cases), and third procedures (six patients). Additional surgery addressed recurrent lateral and far lateral discs (15 patients), recurrent stenosis (15 patients), and a previously undiagnosed neurofibroma (one patient). ${ }^{1}$ The average interval between first and second operations was 49 months (range of 40 months to 7.5 years).

\section{Secondary fusions}

Four of 170 patients undergoing primary far lateral disc surgery developed instability postoperatively and required secondary fusions. All four had far lateral L4-L5 disc herniations in conjunction with Grade I degenerative spondylolisthesis. Original surgical procedures had required full facetectomy thereby resulting in instability. Postoperative instability was documented where a grade I chronic slip and $>4 \mathrm{~mm}$ of active olisthy were demonstrated on dynamic X-ray and myelo-CT studies. The first patient in 1986, was managed with a Hibbs fusion while the three subsequent patients underwent pedicle screw/rod instrumentation. Currently, select individuals may also be candidates for posterior lumbar interbody fusion using cages or bone grafts in conjunction with instrumentation.

\section{Outcomes \\ Patients' outcomes were evaluated postoperatively using a modification of Odom's criteria; excellent (no deficit), good (mild residual radiculopathy requir- ing minimal analgesia), fair (moderate residual radiculopathy or unchanged requiring moderate analgesia), and poor (increased radiculopathy requir- ing increased analgesia). Excellent outcomes were achieved in 73 patients, good outcomes in 51 patients, fair outcomes in 26 patients, and poor outcomes in 20 patients. The frequency of good to excellent outcomes were comparable for all facet-resection techniques utilized; $79 \%$ for the intertransverse approach, $70 \%$ for the full facetectomy and $68 \%$ for the medial facetectomy.}

\section{Common errors in far lateral disc surgery}

Common errors in far lateral disc surgery include operating at the wrong level, making the incorrect diagnosis (ie diabetic amyotrophy), operating on the wrong side, or improper patient selection (prohibitive co-morbidities). A Penfield elevator placed directly into the interspace, not the epidural space while radiographically confirming the level avoids wrong level surgery. Converting full facetectomy-laminectomy technique if there is any technical surgical difficulty provides full visualization of the nerve root laterally, foraminally, and far laterally and will minimize missed fragments and avoid inadvertent root trauma.

\section{Outcome study of far lateral disc surgery using (SF-36)}

Patient-based outcome studies are increasingly utilized to evaluate surgical results and in establishing surgical policy. ${ }^{8}$ The Medical Outcomes Trust Short Form (SF36) is a well-established patient-based outcome instrument which has been successfully employed for over two decades in more than 260 medical and surgical settings. It is easily administered in $15 \mathrm{~min}$ over the telephone or in the office.

The SF-36 measures eight dimensions of outcome; physical function, role physical, bodily pain, general health, vitality, social function, role-emotional and mental health. Thirty-six generic questions are rated on graded scales, raw scores are calculated for each of the eight Health Scales and are then converted to a transformed scale $(0-100 \%)$ (raw score - lowest possible raw score for that health scale, divided by the raw score multiplied by 100). Difference scores also take into account patients' normative data based on age and sex obtained from 2474 individuals in the general US population.

The SF-36 was completed by $76(45 \%)$ of 170 patients in our far lateral disc series from 19841994. ${ }^{1,8,13}$ Interviews were performed over the telephone by one independent interviewer and $100 \%$ of those contacted successfully completed the questionnaire. The average period between the last visit to the surgeon (the time of the surgeon-based assessment) and questionnaire was 2.8 years (standard deviation of 2.3) with a range of 0.6 to 10.2 years. Patients averaged 60 years of age, and included 43 males and 33 females.

The surgeons outcome was based on the patient's last postoperative visit and on Odom's criteria. The 76 patients were last examined by the surgeon an average of 9.1 months following their surgery and were categorized as; excellent ( 32 patients $=42 \%$ ), good $(24=31.5 \%)$, fair $(12=16 \%)$, and poor $(8=11 \%)$ outcomes. The median time between the operation and the surgeon's outcome analysis was 4.5 months (average 9.1 months): $75 \%<9$ months and $90 \%<22$ months), the longest period between surgery and the final clinical assessment was 75 months. There was a statistically insignificant correlation (Spearman rankorder of 0.13) between the surgeon's evaluation and the time since surgery in the direction of better outcome ratings for increased time since surgery.

Modest correlations were established between the surgeon's assessment and SF-36 scores. A positive trend indicated a correlation between the patient's and 
the surgeon's measures except regarding General Health. A significant positive correlation was established between six of the eight SF-36 Health Scales and the surgeon-based assessment. Correlation coefficients using the Spearman rank-order correlation $r$ varied from 0.329 to 0.205 for these six scales; correlation coefficients for General Health and Mental Health were lower (0.088 and 0.160). A difference score was obtained by subtracting the specific age and sex matched norm from the patient's SF-36 transformed score. The mean difference score was then obtained by averaging these data across the eight categories. Although correlations were positive, they were modest in size.

The interval period between the surgeon's assessment and the administration of the SF-36 varied 0.6 to 10.2 years. There was, however, a discrepancy between the patient's self-analysis and the surgeon's evaluation over longer postoperative intervals. A 4.5 year cut-off point was chosen maintaining $75 \%$ (56 patients) of patients in the cohort, while providing a reasonable postoperative time frame. Correlations for this shorter period were larger than those established for all 76 patients followed for up to 10 years varying from 0.187 to 0.378 with all but General Health and Social Function passing statistical significance.

Correlations between the surgeon's assessment and SF-36 responses were modest. For patients seen within the last 4.5 years, the surgeon's assessment was a relatively good predictor of mean SF-36 outcome scores. Physical Function, Role Physical and Bodily Pain best correlated on a descending scale with the surgeon's assessment of physical function.

Future studies should prospectively require that the SF-36 be administered at each postoperative visit for an equal and specific interval and then correlated directly with the surgeon-based evaluations. An openended additional question such as 'Since your surgery has there been any other medical or surgical problem that has interfered with your recovery?' might further enhance the existing SF-36 form.

\section{References}

1 Epstein NE. Evaluation of varied surgical approaches used in the management of 170 far-lateral lumbar disc herniations: indications and results. J Neurosurg 1995; 83: $648-656$.

2 Schlesinger SM, Fankhauser H, de Tribolet N. Microsurgical anatomy and operative technique for extreme lateral lumbar disc herniations. Acta Neurochir (Wien Austria) 1992; 118(3-4): 117-129.

3 Grimes PF, Massie JB, Garfin SH. Anatomic and biomechanical analysis of the lower lumbar foraminal ligaments. Spine 2000; 25(16): 2009 - 2014.

4 An HS et al. Herniated lumbar disc in patients over the age of fifty. J Spinal Disord (US) 1990; 3(2): 143-146.

5 Ebeling U, Reulen HJ. Are there typical localization of lumbar disc herniations? A prospective study. Acta Neurochir (Wien Austria) 1992; 117(3-4): 143-148.
6 Epstein JA, Epstein NE. Lumbar spondylosis and spinal stenosis. In: Wilkins RH, Rengachary SS (eds). Neurosurgery. 2nd edn. New York: McGraw-Hill, 1996; pp $3831-3840$.

7 Epstein NE, Epstein JA. Surgery for spinal stenosis. In: Wiesel SW, Weinstein JN, Herkowitz H, Dvorak J, Bell G (eds). The Lumbar Spine. 2nd edn. Philadelphia: WB Saunders, 1996; pp 737-757.

8 Epstein NE, Hood DC. A comparison of surgeon's assessment to patient's self analysis (Short Form 36) after far lateral lumbar disc surgery: An outcome study. Spine 1997; 22: $2422-2428$.

9 Epstein NE. Decompression in the surgical management of degenerative spondylolisthesis: advantages of a conservative approach in 290 patients. J Spinal Disord 1998; 11(2): 116-122.

10 Epstein NE. Primary fusion for the management of "unstable" degenerative spondylolisthesis. NeuroOrthopedics 1998; 23: $45-52$.

11 Siebner HR, Faulhauer K. Frequency and specific surgical management of far lateral lumbar disc herniations. Acta Neurochir (Wien Austria) 1990; 105(3-4): $124-131$

12 Epstein NE, Epstein JA, Carras R, Hyman R. Far lateral lumbar disc herniations and associated structural abnormalities. An evaluation in 60 patients of the comparative value of CT, MRI, and myelo-CT in diagnosis and management. Spine (US) 1990; 15(6): $534-539$.

13 Epstein NE. Review article: Different surgical approaches to far lateral lumbar disc herniations. $J$ Spinal Disord 1995; 8(5): $383-394$.

14 Epstein NE. Lumbar surgery for 56 limbus fractures, emphasizing non calcified type II lesions. Spine 1992; 17: $1489-1496$.

15 Epstein NE, Epstein JA. Lumbar decompression for spinal stenosis: surgical indications and techniques with and without fusion. In: Frymoyer JW, Ducker TB, Kostuik JP, Whitecloud TS (eds). The Adult Spine. 2nd edn. Philadelphia, New York: Lippincott-Raven, 1997; pp 2055-2088.

16 Epstein BS, Epstein JA, Jones MD. Degenerative spondylolisthesis with an intact neural arch. Radiol Clin North Am 1977; 15: 227 - 239.

17 Winter DD, Munk PL, Helms CA. CT and MR of lateral disc herniation: typical appearance and pitfalls of interpretation. Can Assoc Radiol J (Canada) 1989; 40(5): $256-269$

18 Glickstein MF, Sussman SK. Time-dependent scar enhancement in magnetic resonance imaging of the postoperative lumbar spine. Skeletal Radiol (Germany) 1991; 20(5): 333-337.

19 Garrido E, Connaughton PN. Unilateral facetectomy approach for lateral lumbar disc herniation. J Neurosurg 1992; 76(2): $342-343$.

20 Rust MS, Olivero WC. Far lateral disc herniations: the results of conservative management. J Spinal Disord 1999; 12(2): $138-140$.

21 Weiner BK, Fraser RD. Foraminal injection for lateral lumbar disc herniation. $J$ Bone Joint Surg Br (England) 1997; 79(5): $804-807$.

22 Jane JA, Haworth CS, Broaddus WC. A neurosurgical approach to far-lateral disc herniation. Technical Note. $J$ Neurosurg (US) 1990; 72:(1): $143-144$. 
23 Postacchini F, Cinotti G, Guimna S. Microsurgical excision of lateral lumbar disc herniation through an interlaminar approach. J Bone Jt Surg ( $\mathrm{Br})$ 1998; 80(2): $201-207$.

24 Wiltse LL, Bateman JG, Hutchinson RH. The paraspinal sacrospinalis-splitting approach to the lumbar spine. $J$ Bone Joint Surg 1960; 50A: 919-921.

25 Darden BV et al. Far lateral disc herniations treated by microscopic fragment excision. Techniques and results. Spine 1996; 20(13): 1500-1505.
26 Kambin P, Casey K, Obrien E, Zhou I. Transforaminal arthroscopic decompression of lateral recess stenosis. $J$ Neurosurg 1996; 84(3): 462-471.

27 Ditsworth DA. Endoscopic transforaminal lumbar discectomy and reconfiguration: a postero-lateral approach into the spinal canal. Surg Neurol 1998; 49(6): 588-597. 